\title{
Observation and Quantum Objectivity
}

\begin{abstract}
The paradox of Wigner's friend challenges the objectivity of description in quantum theory. A pragmatist interpretation can meet this challenge by judicious appeal to decoherence. On this interpretation, quantum theory provides situated agents with resources for predicting and explaining what happens in the physical world - not conscious observations of it. Even in Wigner's friend scenarios, differently situated agents agree on the objective content of statements about the values of physical magnitudes. In more realistic circumstances quantum Darwinism also permits differently situated agents equal observational access to evaluate their truth. In this view, quantum theory has nothing to say about consciousness or conscious experiences of observers. But it does prompt us to reexamine the significance even of everyday claims about the physical world.
\end{abstract}

\section{Introduction}

Wigner (1967) presented his "friend paradox" to motivate the view that consciousness has a special role to play in quantum theory-by collapsing the quantum state. In a more recent discussion, D'Espagnat (2005) argued that a consistent treatment of the Wigner's friend scenario may be given (without collapse) if the descriptive content underlying quantum theory is restricted to probabilistic predictions flowing from the Born Rule, but only if these concern conscious experiences of observers. In contrast, a recent pragmatist proposal (Healey 2012) views quantum theory as a resource for situated agents (rather than observers) with no requirement that these be conscious 11 If quantum theory is interpreted along these pragmatist lines, Wigner's friend scenarios may be treated consistently and without ambiguity.

The paper proceeds as follows. Section 2 analyzes distinct notions of objectivity relevant to quantum theory. Section 3 recalls the paradox of Wigner's friend. The pragmatist interpretation of Healey (2012) is sketched in section 4. Section 5 shows how this resolves the paradox while securing the objectivity of description in quantum theory. Section 6 addresses a more general worry about objectivity of description. Section 7 relates the foregoing treatment of objectivity to a recent suggestion (Ollivier et al. 2004) that objective properties emerge from subjective quantum states through quantum Darwinism. While a pragmatist view of quantum theory secures the objectivity of a claim about the values of physical magnitudes, it recognizes that its content depends on the environmental context. The conclusion points out that this involves a subtle

\footnotetext{
${ }^{1}$ As far as we know, conscious humans are the only agents currently able to avail themselves of this resource. But this view admits the possibility that non-human, or even non-conscious, situated agents may come to use quantum theory.
} 
change in our understanding of how even the familiar language of everyday affairs, including laboratory procedures, assigns objective properties-beables-to objects (Bell 2004).

\section{Objectivity}

Objectivity (and its polar opposite, subjectivity) can mean many things. Dreams are a paradigm of subjectivity. The subject matter of a dream is not objective: a dream does not portray what really happens. The mode of presentation is not objective: this subject matter is accessible only to the dreamer. What is presented as well as its mode of presentation strongly depends on the specific as well as the general features of the dreamer-her individual physiology, psychology and prior experiences as well as her humanity (Dick 1968). By contrast, a description or mathematical representation in physics has an objective subject matter if its content represents physical reality, and an objective mode of presentation to the extent that how this is represented does not depend on the specific and general features of the one whose representation it is.

The prominence of notions of observation or measurement in standard formulations of quantum theory raises concerns about the objectivity of descriptions in that theory. If the Born Rule is understood to yield probabilities just for results of observations/measurements then one can question the objectivity of these results. The orthodox view - that a quantum measurement cannot be understood generally as revealing the value of the measured observable - has now been amply supported by "no-go" theorems (Gleason 1957, Kochen and Specker 1967, Fine 1982, Mermin 1993). This challenges the objectivity of subject matter in a description of the result of an observation. One can respond to this challenge by proposing an analysis of observation/measurement as an objective physical process. But the assumption that quantum theory can itself represent this process leads to the notorious quantum measurement problem (Fine 1970, Brown 1986, Bassi and Ghirardi 2000). The alternative of characterizing observation independently as a physical interaction with a macroscopic apparatus, and/or involving irreversible amplification involves the "shifty split" between quantum and classical descriptions justifiably criticized in Bell (2004).

With his keen appreciation of how difficult it is to provide a satisfactory physical characterization of observation/measurement in quantum theory, Wigner came to think that a measurement in quantum theory occurs just when a conscious observer becomes aware of a result. On this view, the Born Rule yields probabilities only for alternative conscious experiences. It thereby threatens both the objectivity of the subject matter of description in quantum theory and the objectivity of its mode of presentation. Quantum theory, it seems, is then concerned to predict and (perhaps) explain "communicable human experience. In other words...the set of all the impressions human minds may have and communicate to others." (D'Espagnat 2005) 2

\footnotetext{
${ }^{2}$ The Wigner's friend scenario casts doubt even on such communicability. D'Espagnat's paper seeks to relieve this doubt.
} 
Suppose one so restricts the scope of quantum theory. Then the descriptive claims to which the Born Rule attaches probabilities fail to be objective in several respects:

(1) Their subject matter is not a physical reality independent of our experiences.

(2) Their mode of presentation depends on the individual consciousness.

(3) This consciousness is generically human.

One could seek to restore this third respect of objectivity by generalizing beyond humans to observers capable of any sufficiently sophisticated form of conscious experience. But since consciousness is not well understood (especially in nonhumans) to do so would further obscure the content of these descriptive claims, which would still fail to be objective in either of the first two respects.

While the quantum theory of environmental decoherence does not by itself resolve the quantum measurement problem (D'Espagnat 1990, Bub 1997, Adler 2000), many believe it may contribute to a resolution within some appropriate interpretative framework. Such a framework appears in (Healey 2012), and is sketched in section 4 below. From the present perspective, the key interpretative proposal is to remove any talk of observation or measurement from a formulation of the Born Rule by taking this to generate mathematical probability distributions over claims that simply describe values of magnitudes rather than results of observing them. The "no-go" theorems block this approach if one further assumes that every claim over which these mathematical distributions are defined always has a well-defined content, so an agent should believe it to a degree specified by its Born "probability" to avoid refutation by statistics of actual observations. But suppose, on the other hand, that how much significance attaches to a claim about a system depends on the extent of environmental decoherence suffered by its quantum state. Then the numbers yielded by the Born rule have the import of genuine probabilities only for significant claims: Born rule "probabilities" for claims lacking such significance do not correspond to (actual or hypothetical) frequencies, and should not guide an agent's degrees of belief in these insignificant claims.

On this view any reference to observation or measurement has been eliminated from the Born rule as well as other basic principles of quantum theory, and so there is no reason to suppose that descriptive claims that arise in quantum theory are subjective in any of the respects (1)-(3) noted above. But there is a fourth aspect of objectivity to consider, as the following quote makes clear.

A view or form of thought is more objective than another if it relies less on the specifics of the individual's makeup and position in the world, or on the character of the particular type of creature that he is. (Nagel 1986)

If the significance for an agent of a descriptive claim about the value of a magnitude depends on how that agent is situated in the world, then that claim may lack a kind of objectivity. Differently positioned agents could understand the claim differently: they may come to a no-fault disagreement about whether 
it is true, or even meaningful. The interpretation of Healey (2012) faces such a challenge to its understanding of the objectivity of descriptive claims in quantum theory - a challenge that is highlighted by the Wigner's friend scenario, as we shall see.

\section{Wigner's friend}

The "paradox" of Wigner's friend presents a challenge to the objectivity of physical description within quantum theory. To set up the "paradox", imagine Schrödinger's cat (and associated 'diabolical device') replaced by a human experimenter (Wigner's friend, John) who records in a device $D$ the result of a quantum measurement of observable $Q$ he performs on a system $s$ inside his isolated laboratory 3 Eugene and John both agree that the quantum state of $s$ is initially (at time $t_{i}$ ) a non-trivial superposition of eigenstates of $Q$. Let $C$ be the claim that the value of a recording magnitude $M$ on $D$ is $m$ at time $t_{f}$ after $s$ has interacted with $D$. John looks at $D$ at $t_{f}$ and makes claim $C$. On the basis of this observation, he assigns a "collapsed" quantum state to $D+s 4$ Environmental interactions within the laboratory rapidly entangle this "collapsed" state with the state of everything else in the laboratory. But the total quantum state $\left|\psi_{J}\right\rangle$ of the enormously complex system composed of John, $D$ and everything else inside the laboratory will continue to reflect the "collapse" onto an eigenstate of $M$ induced by John's measurement.

Meanwhile Eugene, who has remained outside the laboratory, assigns a state $\left|\psi_{E}\right\rangle$ to the enormously complex system composed of John, $D$ and everything else inside the laboratory, based on all the information about the properties of systems to which he has access in this situation. For Eugene, John's measurement involves purely unitary interactions - between $s$ and $D$, between $D$ and John, and between all these systems and the rest of the laboratory. Accordingly, he evolves the state $\left|\psi_{E}\right\rangle$ unitarily, with no "collapse" from $t_{i}$ to $t_{f}$. Since $\left|\psi_{J}\left(t_{f}\right)\right\rangle \neq\left|\psi_{E}\left(t_{f}\right)\right\rangle$, John's and Eugene's quantum states differ after John's measurement but before Eugene enters the laboratory. As these states are usually understood, $\left|\psi_{J}\left(t_{f}\right)\right\rangle$ represents a definite result of John's measurement (recorded by the state of $D$, John's memory, etc.) while $\left|\psi_{E}\left(t_{f}\right)\right\rangle$ excludes any such definite result and its traces. To retain its internal consistency, this view of quantum states must deny the objectivity of John's measurement result, since differently situated agents (John and Eugene) disagree about whether $C$ is true at $t_{f}$.

\footnotetext{
${ }^{3}$ To call the laboratory 'isolated', is to require by fiat the absence of any decohering interactions with its external environment. So we are talking of a ridiculously impractical Gedankenexperiment, as Schrödinger explicitly said he was when describing his cat scenario. The point of doing so is to show how this raises a problem for one view of quantum theory and then to explain why this problem does not arise on the pragmatist view of Healey (2012).

${ }^{4} \mathrm{He}$ does so in accordance with a view of quantum states due to Dirac and von Neumann. While himself subscribing to this orthodoxy, Wigner maintained that it is only John's consciousness that induces the collapse.
} 
On any view of quantum states, since $\left|\psi_{E}\left(t_{f}\right)\right\rangle$ is an eigenstate of some extremely complex observable $O$ on the entire laboratory system, Eugene could in principle (though certainly not in practice) distinguish between $\left|\psi_{J}\left(t_{f}\right)\right\rangle$ and $\left|\psi_{E}\left(t_{f}\right)\right\rangle$ by creating a suitable ensemble of identical laboratory systems and measuring $O$ on each system: $\left|\psi_{E}\left(t_{f}\right)\right\rangle$ would give the same result on each, while $\left|\psi_{J}\left(t_{f}\right)\right\rangle$ would almost certainly yield a statistical spread of different results. This same procedure would also, in principle, enable Eugene to distinguish between $\left|\psi_{E}\left(t_{f}\right)\right\rangle$ and the mixed state $\rho_{J}\left(t_{f}\right)=\sum_{i}\left|c_{i}\right|^{2}\left|\psi_{J}\left(t_{f}\right)\right\rangle_{i}\left\langle\left.\psi_{J}\left(t_{f}\right)\right|_{i}\right.$ Eugene may assign to reflect his ignorance of the result of John's measurement 5

Wigner's own way of resolving this paradox was to give consciousness (and only consciousness) the distinctive physical role of inducing "collapse" of the quantum state onto an eigenstate of the measured observable. For him, it was the interaction with John's consciousness that produced a discontinuous physical change inside the laboratory, resulting in the final state $\left|\psi_{J}\left(t_{f}\right)\right\rangle$ and not $\left|\psi_{E}\left(t_{f}\right)\right\rangle$. Such a change would be detectable in principle in the Wigner's friend scenario, though quite impossible to detect in practice. I will offer a different resolution involving no such physical "collapse". This involves the different understanding of quantum states described in the next section.

\section{A pragmatist interpretation}

In quantum theory as it is usually formulated, theoretical models involve quantum states and operators corresponding to observables (including the Hamiltonian and/or Lagrangian) and (solutions to) the Schrödinger equation and relativistic generalizations. But there is still no agreement as to how, or whether, any of these model elements represent physical magnitudes.

According to Healey (2012), observables, quantum states and their evolution neither represent nor describe the condition or behavior of any physical system. It follows that quantum theory does not imply statements one can use to make claims about natural phenomena that describe or represent features of those phenomena. But quantum theory nevertheless helps us to predict and explain an extraordinary variety of physical phenomena using representational resources from outside of quantum theory. It can do this because of the way in which theoretical models involving quantum states, operators, and the Schrödinger equation are applied. There are principles for using these models to guide one in making descriptive claims and forming representational beliefs about physical systems to which such models may be applied but which these models do not themselves describe or represent.

The dispute as to whether quantum theory provides a complete description of a physical system presupposes that quantum states at least provide a partial description or representation of the physical properties of systems to which they are assigned. Rejecting this presupposition may seem tantamount

\footnotetext{
${ }^{5}$ To keep things simple, here I assume John made an ideal measurement on the state $\sum_{i} c_{i}\left|\varphi_{i}\right\rangle$, thereby collapsing the state of $s+D$ onto some eigenstate $\left|\varphi_{j}\right\rangle\left|\psi_{j}\right\rangle$ with probability $\left|c_{j}\right|^{2}$.
} 
to regarding the quantum state as merely a symbolic device for calculating probabilities of possible measurement outcomes on these systems. But this is not so. Assignment of a quantum state may be viewed as merely the first step in a procedure that licenses a user of quantum theory to express claims about physical systems in descriptive language and then warrants that user in adopting appropriate epistemic attitudes toward these claims. The language in which such claims are expressed is not the language of quantum states or operators, and the claims are not about probabilities or measurement results: they are about the values of magnitudes. That is why Healey (2012) refers to such claims as NQMC's - Non-Quantum Magnitude Claims. Here are some typical examples of NQMC's:

A helium atom with energy -24.6 electron volts has zero angular momentum.

Silver atoms emerging from a Stern-Gerlach device each have angularmomentum component either $+\hbar / 2$ or $-\hbar / 2$ in the $z$-direction.

The fourth photon will strike the left-hand side of the screen.

When a constant voltage $V$ is applied across a Josephson junction, an alternating current $I$ with frequency $2(e / h) V$ flows across the junction.

(Notice that two of these non-quantum claims are stated in terms of Planck's constant.) For contrast, here are some quantum claims which do not describe the physical properties of systems to which they pertain:

The expectation value of angular momentum for atomic helium in the ground state is 0 .

Integral-spin systems have symmetric quantum states, while halfintegral-spin systems have antisymmetric quantum states.

The probability that a tritium nucleus will decay in 12.3 years is $\frac{1}{2}$.

After one photon from the polarization-entangled Bell state $|\Phi+\rangle$ is found to be horizontally polarized, the other photon has polarization state $\mid \mathrm{H}>$.

This is not to say that quantum mechanical claims like these lack truthvalues - each is appropriately evaluated as objectively true (though in the case of the last claim that evaluation is critically dependent on the context relative to which it is made). But the function of such claims is not to describe or represent properties of physical systems: it is to offer authoritative advice to a physically situated agent on the content and credibility of NQMC's concerning them. Quantum theory contributes indirectly to our predictive and explanatory projects.

A quantum state and the consequent Born probabilities can be assigned to a system only relative to the physical situation of an (actual or hypothetical) agent for whom these assignments would yield good epistemic advice. What 
one agent should believe may be quite different from what another agent in a different physical, and therefore epistemic, situation should find credible. This relational character of quantum states and Born probabilities does not make these subjective, and it may be neglected whenever users of quantum theory find themselves in relevantly similar physical situations. NQMC's are not relational in this way: Their truth-values do not depend on the physical situation of any actual or hypothetical agent.

On this pragmatist understanding, a quantum state guides an agent in two different ways. The agent requires guidance in assessing the content of NQMC's about systems of interest in a context where such claims may arise. It is often said that assignment of a value to an observable on a system is meaningful only in the presence of some apparatus capable of measuring the value of that observable. But some account of meaning must be offered in support of this assertion, and the extreme operationist account that is most naturally associated with it would be unacceptably vague even if it were otherwise defensible. Exactly what counts as the presence of an apparatus capable of measuring the value of an observable?

Contemporary pragmatist approaches to meaning have the resources to provide a better account of the significance of a NQMC about a system, as entertained by an agent, in a context in which that system features. A pragmatist like Brandom $(1994,2000)$ takes the content of any claim to be articulated by the material inferences (practical as well as theoretical) in which it may figure as premise or conclusion. These inferences may vary with the context in which a claim arises, so the content of the claim depends on that context. The quantum state modulates the content of NQMC's about a system by specifying the context in which they arise. The context may be specified by the nature and degree of environmental decoherence suffered by this quantum state. A NQMC about a system when the quantum state has extensively decohered in a basis of eigenstates of the operator corresponding to that magnitude on the system has a correspondingly well-defined meaning: a rich content accrues to it via the large variety of material inferences that may legitimately be drawn to and from the NQMC in that context. Call a NQMC canonical if it is of the form $M \in \Delta$ :

Magnitude $M$ has value in Borel set $\Delta$ of real numbers.

Only when the content of a canonical NQMC is sufficiently well articulated in this way is it appropriate to apply the Born Rule to assign a probability to that claim.

For example, a claim that an electron passed unobservably through a particular slit in a diffraction grating figures as premise or conclusion in almost no interesting material inferences, and so is very poorly articulated. This is a consequence of the fact that, in the absence of interactions capable of revealing its presence, the quantum state of the electron suffers negligible decoherence through entanglement with the environment in the interferometer. With no significant decoherence between different spatially localized quantum states, an agent has only a very limited license to use claims about the electron's position. 
In particular, the license is so limited that little may legitimately be inferred from such claims. But the subsequent interaction with detectors at the screen involves massive environmentally induced decoherence of the initial quantum state of electron plus detector, permitting a high degree of articulation of the content of claims about the value of a magnitude on the detector taken to record the position of the electron at the screen. The decohered quantum state then grants an agent a wide license to formulate and use NQMC's about records of the electron's position at the screen. This illustrates the first way in which a quantum state guides an agent - by advising her on the extent of her license to use particular NQMC's by informing her of the nature and degree of that state's environmental decoherence.

With a sufficiently extended license, an agent may now apply the Born Rule to evaluate the probability of each licensed canonical NQMC using the appropriate quantum state. In the example, this will be the initial superposed state of the electron. Lacking more direct observational information, after assigning the quantum state of a system appropriate to her physical situation, an agent should adjust her degrees of belief in licensed NQMC's pertaining to that system so they match the probabilities of NQMC's specified by the Born Rule. This is the second way in which a quantum state guides an agent.

An agent should accept this twofold guidance by the quantum state appropriate for one in her physical situation, since by doing so she is able successfully to predict and explain what happens in a wide variety of circumstances. But according to Healey (2012) she need not, and should not, take this quantum state to describe or represent ontological features of a system to which she ascribes it. The interpretation does not deny that environmental decoherence involves a physical process. But it does deny that decoherence of the quantum state represents changes in intrinsic properties of a physical system. An agent appeals to the quantum theory of decoherence to decide what to think about the content and credibility of NQMCs about a physical system, not to describe the evolving properties of a system whose quantum state decoheres.

\section{Paradox resolved}

Consider the situation of Wigner's friend John inside his isolated laboratory. According to the pragmatist interpretation sketched in the previous section, John is licensed by decoherence to apply the Born Rule to the superposed state of $s$ and advised by quantum theory to adjust his credences in claims about the value of recording magnitude $M$ on $D$ at time $t_{f}$ so they match the corresponding Born probabilities. This is because the phase of the quantum state he consequently assigned to $D+s$ immediately before has, by $t_{f}$, been robustly delocalized into the environment inside his laboratory. He is then warranted by his own direct observation of $D$ in making the significant claim $C$ that the value of $M$ on $D$ is $m$.

Meanwhile Eugene, who has remained outside the laboratory, assigns state $\left|\psi_{E}\right\rangle$ to the enormously complex system composed of John, $D$ and everything 
else in the laboratory. By assumption, the phase of state $\left|\psi_{E}\right\rangle$ has not been delocalized into its environment by $t_{f}$, so this state does not license Eugene to entertain any significant claim about the value of a magnitude on the laboratory or anything in it. In this situation, Eugene should not apply the Born Rule to $\left|\psi_{E}\right\rangle$ and adjust any of his credences accordingly. If and only if the laboratory ceases to be isolated so as to delocalize the phase of $\left|\psi_{E}\right\rangle$ (as Eugene subsequently enters it, for example) would Eugene be in a position to apply the Born Rule to significant claims about the laboratory or traces it leaves in an external recording device (such as his own brain).

Recall the difficulties presented by the Wigner's friend scenario noted in section 3:

1) As these states are usually understood, $\left|\psi_{J}\left(t_{f}\right)\right\rangle$ represents a definite result of John's measurement (recorded by the state of $D$, John's memory, etc.) while $\left|\psi_{E}\left(t_{f}\right)\right\rangle$ excludes any such definite result and traces of it in John's laboratory.

2) On any view of quantum states, since $\left|\psi_{E}\left(t_{f}\right)\right\rangle$ is an eigenstate of some extremely complex observable $O$ on the entire laboratory system, Eugene could in principle (though certainly not in practice) distinguish between $\left|\psi_{E}\left(t_{f}\right)\right\rangle$ and $\left|\psi_{J}\left(t_{f}\right)\right\rangle$ (or $\left.\rho_{J}\left(t_{f}\right)\right)$ by creating a suitable ensemble of identical laboratory systems and measuring $O$ on each system.

These difficulties present a challenge to the objectivity of physical description here. (1) apparently implies that, by assigning different quantum states to the contents of John's laboratory at $t_{f}$, John and Eugene come to disagree about the truth-value of the descriptive claim $C$. If so, they can both be right only if that truth-value is relative to the physical situation of the agent making it - in conflict with the fourth aspect of objectivity noted in section 2 . But (2) shows that such relativization of truth-value to agent-situation still leaves it unclear how John and Eugene can consistently apply quantum theory here. Independent of the practicality of Eugene's discriminatory measurements, $\left|\psi_{E}\left(t_{f}\right)\right\rangle$ and $\left|\psi_{J}\left(t_{f}\right)\right\rangle$ (or $\left.\rho_{J}\left(t_{f}\right)\right)$ are distinct states, yielding incompatible Born probabilities concerning the possible values of certain magnitudes on the entire laboratory. Relativization of the laboratory's quantum state to the situations of Eugene, John, respectively leaves it ambiguous on which of these states quantum theory advises them to base their expectations about these possible values.

The pragmatist of the previous section responds to this challenge, first by rejecting the usual understanding of the states $\left|\psi_{J}\left(t_{f}\right)\right\rangle,\left|\psi_{E}\left(t_{f}\right)\right\rangle$. For her, neither state has the function of representing the physical properties of John's laboratory or anything in it. Since neither state represents anything bearing on the result of John's measurement (e.g. whether or not $C$ is true at $t_{f}$ ), assignment of both states to John's laboratory at the same time could not lead John and Eugene to assign (apparently) conflicting truth-values to $C$.

But John and Eugene each assume that John will perform a measurement on $s$ by interacting $s$ appropriately with $D$, and that the environment inside the laboratory will decohere the state of $D+s$ in $D$ 's "pointer basis" (without inducing any physical collapse in that state). So both John and Eugene are licensed to entertain claim $C$ and (beforehand) to set their credences for $C$ at $t_{f}$ equal to the corresponding Born Rule probability. This implies they agree 
that $C$ has a truth-value at $t_{f}$. But while Eugene remains outside the laboratory, only John is in a position to look at $D$ at and after $t_{f}$ and so determine what that truth-value is. This secures the objectivity of the description $C$, in the sense that differently positioned agents (John and Eugene, in this case) agree that $C$ has a truth-value after $t_{f}$, and do not disagree about what that truth-value is. At this stage, John, but not Eugene, is in a position to know that $C$ is true rather than false. Eugene can choose whether or not to enter the laboratory to try to find out whether $C$ is true.

Suppose Eugene decides to see for himself the outcome of John's observation by entering the laboratory just after $t_{f}$. This will decohere the phase of $\left|\psi_{E}\left(t_{f}\right)\right\rangle$, permitting Eugene to apply the Born Rule to this state to adjust his degrees of belief in various significant claims about the contents of the laboratory, including $C$, John's record of the outcome in his notebook, John's verbal report, etc. as well as correlations between such claims. The Born probabilities of these claims (joint as well as single) based on the state $\left|\psi_{E}\left(t_{f}\right)\right\rangle$ will lead him to the following confident expectation: whatever may be the outcome of John's observation, the laboratory will contain multiple mutually supporting records of it. Nothing about $\left|\psi_{E}\left(t_{f}\right)\right\rangle$ will tell him what John's outcome actually was. But he can easily find that out by asking John and observing any of the other multiple, correlated records inside the laboratory. The important point is that $\left|\psi_{E}\left(t_{f}\right)\right\rangle$ does not exclude an outcome at $t_{f}$, so by entering the laboratory Eugene simply finds out what happened-he does not make it happen.

If Eugene were instead (able) to remain outside the laboratory but interact with it so as to measure the value of $O$, he should use $\left|\psi_{E}\left(t_{f}\right)\right\rangle$ to calculate a Born probability of 1 that a suitable recording device, applied to the laboratory and decohered in its "pointer basis" by external environmental interactions, will record a value of $O$ equal to the eigenvalue of its corresponding operator in state $\left|\psi_{E}\left(t_{f}\right)\right\rangle$. Even though Eugene assumes that John records the outcome of his measurement at $t_{f}$, by $t_{f}$ there has been no physical interaction between Eugene and anything inside the laboratory that could serve to inform Eugene of that outcome. So Eugene cannot base his expectation of the outcome of a measurement of $O$ on $\left|\psi_{J}\left(t_{f}\right)\right\rangle$, and would be mistaken if he were to base that expectation on $\rho_{J}\left(t_{f}\right)$. There is no ambiguity about to what state Eugene should apply the Born Rule when setting his credences concerning the outcome of his $O$ measurement.

On what quantum state should John base his credence as to the outcome of an external $O$-measurement? The outcome of John's measurement should prompt him to update his quantum state for objects in his laboratory. These include $D, s$ and other systems inside the laboratory, but not himself, considered as a physical system: the function of John's quantum state is to set his credences concerning claims about these other systems, but not about himself. John should not base his credences as to the outcome of an external $O$ measurement on either of the states $\left|\psi_{J}\left(t_{f}\right)\right\rangle$ or $\rho_{J}\left(t_{f}\right)$, since these are states of himself as well as everything else in his laboratory. He can hypothetically take the "external" perspective of Eugene, but if he does so he must then assign the same state $\left|\psi_{E}\left(t_{f}\right)\right\rangle$ that he agrees is the correct state for someone in Eugene's 
situation. There is no ambiguity as to which quantum state should be used to calculate Born probabilities for the results of external measurements performed on the entire laboratory, in the sense that differently positioned agents (John and Eugene, in this case) agree on this state. Of course, only Eugene is then in a position to make such a measurement.

What will Eugene find if he enters the laboratory after measuring the value of $O$ in this way? $D$ will no longer have a record of the truth of $C$. Even if John still exists, he will not remember that $C$ was verified as true at $t_{f}$. Because $\hat{O}$ fails to commute with the operator corresponding to $M$, as well as an effective "pointer observable" on every other system correlated with this through interactions within the laboratory, the external interaction required to record the value of $O$ will have so disturbed the laboratory and its contents as to remove all traces that $C$ was true at $t_{f}$. But one can't change the past: $C$ was indeed true at $t_{f}$, as John then verified with his own eyes.

There is, in principle, an even more dramatic way to erase all traces of $C$. Since the entire laboratory and its contents constitutes an isolated system, Eugene will take $\left|\psi_{E}\right\rangle$ to have evolved unitarily from its state $\left|\psi_{E}\left(t_{i}\right)\right\rangle$ prior to John's measurement on $s$ to its state $\left|\psi_{E}\left(t_{f}\right)\right\rangle=U_{i f}\left|\psi_{E}\left(t_{i}\right)\right\rangle$. The state Eugene should ascribe to the contents of the laboratory at $t_{i}$ should reflect his belief that his friend has not yet performed the planned measurement: this will assign Born probability 1 to claims about $D$, John and other items in the laboratory that suffices to substantiate this full belief. Mathematically, there will exist a Hamiltonian that would induce the time-reversed evolution of $\left|\psi_{E}\right\rangle$ so that at a later time $t_{g}$ (where $t_{g}-t_{f}=t_{f}-t_{i}$ ) it is restored to its value before John's measurement: $\left|\psi_{E}\left(t_{g}\right)\right\rangle=U_{f g}^{\dagger}\left|\psi_{E}\left(t_{f}\right)\right\rangle=\left|\psi_{E}\left(t_{i}\right)\right\rangle$.

If Eugene had the powers of a quantum demon, he could instantaneously replace the original Hamiltonian by this time-reversing Hamiltonian at $t_{f}$, thereby restoring $\left|\psi_{E}\right\rangle$ at $t_{g}$ to its original value at $t_{i}$. Suppose that he does so, and postpones his entry into the laboratory until $t_{g}$. Since the quantum state of the entire laboratory is identical to what it was before John had made any measurement, Eugene must fully expect that if he then asks John about the result of his measurement, John will say he has not yet performed any measurement. He must further fully expect that his own examination at, and at any time after, $t_{g}$ of $D$, John's notebook, and anything else inside the laboratory will reveal no record of any such measurement ever having been made. Eugene's action at $t_{f}$ has, by $t_{g}$, erased all traces of John's measurement and its result: Indeed, Eugene has succeeded in erasing all traces of everything that happened inside the laboratory between $t_{i}$ and $t_{f}$. Once again, he has not changed the past. But there has been a wholesale loss of history, understood as reliable information about the past. This point will be pursued in the concluding discussion. The present section has shown how the pragmatist view of Healey (2012) maintains the objectivity of physical description in the Wigner's friend scenario. 


\section{Objectivity secured}

According to Healey (2012), John inside the laboratory and Eugene outside do not assign inconsistent truth-values to claim $C$. Moreover, each assigns the same rich content to $C$ based on the decoherence of the state of $s+D$ induced by environmental interactions inside the laboratory. This certifies the objectivity of $C$ 's content in the Wigner's friend scenario. The certification depends on the fact that both John and Eugene apply essentially the same model of decoherence to the same initial quantum state of $s+D$.

But, according to Healey (2012), while quantum states are objective they are also relational: the quantum state an agent should assign to a system generally depends on the (actual or hypothetical) physical situation of that agent. If differently situated agents assign different quantum states to a system, this raises the possibility that their models of its decoherence will so differ as to lead each to assign a different content to certain claims concerning it. Relativization of quantum state ascriptions threatens relativization of the content of NQMCs like $C$. This would undermine their objectivity in the fourth respect pointed out in section 2 .

Now even though John and Eugene are differently situated in the Wigner's friend scenario, each can choose to adopt the perspective of the other's situation for the purpose of applying quantum theory. Eugene should assign the same initial state as John to $s$ because each knows that this is the state on which John will perform his measurement. Eugene and John are each in the same state of ignorance as to the initial state of $D$ and its (lack of) correlations with that of $s$, so there is no reason for them to assign different states either to $D$ alone or to $s+D$. (Their conclusion - that interactions within the laboratory robustly decohere the state of $s+D$ and so endow $C$ with rich content-is not sensitive to fine details of the initial state of $s+D$.)

One might object that Eugene's physical situation requires him to assign the state $\left|\psi_{E}\right\rangle$ to the contents of the laboratory, and that since the phase of $\left|\psi_{E}\right\rangle$ is not delocalized into its environment Eugene should not assign a rich content to $C$. An alternative objection would be that $C$ has no unambiguous content for Eugene, since he has no principled reason to base his assessment of that content on the degree of decoherence of the "internal" state of $s+D$ rather than that of $\left|\psi_{E}\right\rangle$.

But in assessing the content of an NQMC such as $C$ about a system ( $D$ in this case), an agent like Eugene should base his model of decoherence on quantum state assignments incorporating everything he is in a position to know about the physical situation of $D$, as represented in non-quantum claims. We have assumed that Eugene is in a position to know that John will initiate a certain interaction between $s$ and $D$ inside the laboratory. Eugene's physical situation does not require him to assign the state $\left|\psi_{E}\right\rangle$ to the contents of the laboratory when assessing the content of $C$ : to do so would be to neglect information to which he has access in this situation that is relevant to assignment of quantum states to $s$ and $D$.

There are circumstances in which agents are so differently situated that they 
correctly assign different quantum states to the same system, where each agent's assignment is based on all information to which the agent has access, given her physical situation 6 If models of decoherence based on such different assignments were to result in these agents assigning different contents to the same NQMC, that could threaten the objectivity of description in quantum theory. To assess the threat we need to specify how far different agents' state assignments may differ, and how this affects the models of decoherence they should employ in assessing the content of relevant NQMCs.

The example in footnote 6 prompts the following restriction on different agents' state assignments: If Alice assigns a mixed state to a system while Bob assigns a pure state, then Bob's state vector lies in the support of Alice's density matrix. An argument for a generalization of this restriction has been offered (Brun et al. 2002): Different agents' state assignments by several density matrices are mutually compatible if and only if the supports of all of them have a least one vector in common.

Suppose differently situated agents (such as Alice and Bob in the example) assign quantum states to a system $S$ that differ in some way consistent with this generalized restriction. Assuming this is the only difference between the models of decoherence they use in assessing the content of NQMCs about $S$, there are no grounds for thinking they will arrive at different assessments. By assumption, all their models share the generic features of applying the same Hamiltonians to the same initial state of $S$ 's environment with the same limitations on prior system/environment entanglement. Since a model with these generic features will decohere the phase of a generic pure state of $S$ to the same extent in the same "basis"7, that is how it will model the decoherence of every vector in the Hilbert space in which lies the vector common to the supports of all the states assigned by differently situated agents subject to the generalized restriction. It follows that the model of decoherence each of these agents uses to assess the content of NQMCs about $S$ will lead each agent to the same assessment of the contents of NQMCs about $S$. This means they will all agree on the content of these NQMCs, thereby securing their objectivity.

\section{Independent Verifiability}

It is ironic that observation poses a threat to the objectivity of physical description in quantum theory, since observation is generally used to settle questions about objectivity in science and daily life. Doubting the objective presence of the dagger he saw, Macbeth tried to grasp it: to prove that Banquo's ghost occupied his own place at table, he pleaded his guests to see for themselves. Classical physics permits multiple, independent observations on a system to

\footnotetext{
${ }^{6}$ This happens, for example, when Alice and Bob perform spacelike separated polarization measurements on an entangled photon pair. Knowing his outcome, Bob can assign a polarization eigenstate to the photon entering Alice's detector. Since this information is not accessible to Alice, she correctly assigns that photon a mixed polarization state.

${ }^{7}$ The scare quotes mark the need to allow for models in which decoherence effectively "diagonalizes" s's density operator in an overcomplete basis of narrow Gaussian states.
} 
verify a claim about its state, since none of these need irremediably disturb that state.

But suppose an individual system is in a wholly unknown quantum state. No single observation on it can reliably disclose its state. Repeated observations are no better, since observing a system typically irreparably disturbs its quantum state. So even if a system's wholly unknown quantum state could be ascertained by a single observation, this finding could not be checked in further observations, either by the original agent or by others. A wholly unknown quantum state of an individual system is not as objective as a corresponding classical state in the sense that it is not independently verifiable. Ollivier et al. (2004) put the point like this:

The key feature distinguishing the classical realm from the quantum substrate is its objective existence.

They propose what they call an operational definition of objectivity for a property of a quantum system, according to which such a property is simultaneously accessible to many observers who are able to find out what it is without prior knowledge and who can arrive at a consensus about it without prior agreement. Their idea is that such a property is objective to the extent that multiple records of it exist in separate portions of the environment, so that "observers probing fractions of the environment can act as if the system had a state of its own - an objective state." They say that

The existence of an objective property requires the presence of its complete and redundant imprint in the environment as necessary and sufficient conditions.

While Ollivier et al. (2004) never say exactly what they mean by a property, it seems clear they would count an NQMC locating a value in $\Delta$ of a magnitude $M$ on system $S$ as a property assignment to $S$.

The state of a system in classical physics is specified by a point in phase space: this is equivalent to an assignment of a value to each magnitude on that system, i.e. an assignment of a property locating that value in a unit set. The authors' stand-in for the objective state of an open quantum system is one of the eigenvectors of the system observable corresponding to the pointer magnitude that is selected by environmental decoherence. A NQMC locating a value in $\Delta$ to the pointer magnitude on system $S$ is taken to assign $S$ an objective property solely on the grounds that a complete and redundant imprint is present in the environment. There are other magnitudes on $S$ represented by operators each of whose eigenvectors is "close" to an eigenvector of the pointer observable. A NQMC locating a value in $\Delta$ to such a magnitude on system $S$ also counts as assigning $S$ a reasonably objective property because of a complete and (slightly less) redundant imprint in the environment. Their idea seems to be that the proliferation of imprints of properties of a quantum system in its environment progressively objectifies its properties until these come to mimic properties that characterize a classical state. An eigenvector of the pointer observable stands in 
for an objective state by specifying what properties of the system are objectified by the environmental interactions to which it is subjected.

...amplification of a preferred observable happens almost as inevitably as decoherence, and leads to objective classical reality.

This is Quantum Darwinism: "the idea that the perceived classical reality is a consequence of the selective proliferation of information about the system". It is not an account of classical reality, or of the actual objective state of a quantum system. It is an account of how independent acts of observation on a system's environment can produce consensus on properties of a quantum system irrespective of whether or not that system has such properties. One is reminded of Wittgenstein's remark in his Philosophical Investigations

As if someone were to buy several copies of the morning paper to assure himself that what it said was true.

Wittgenstein's avid reader is actually in better shape than multiple quantum observers. The morning paper may have correctly reported what happened. But if no NQMCs about a quantum system are true then it lacks the properties observers attribute to it, so whatever proliferates is in fact misinformation. It is important not to be misled by the causal language of imprints into thinking that objective properties of the environment are caused by objective properties of a decohered quantum system. As it stands, Quantum Darwinism (Ollivier et al. 2004) fails to provide an adequate account of objectivity in the sense of the independent verifiability of NQMCs because it does nothing to show how those NQMCs can be objectively true, given the quantum states of system and environment. Groupthink does not amount to intersubjective verification. But the pragmatist view of Healey (2012) can help the quantum Darwinist take this crucial last step.

In that view, decoherence endows certain NCMCs with a significant content that requires even differently situated agents to seek agreement on their truthvalues. When a system is decohered by its environment, these will include claims about the value of its pointer magnitude. They will also include claims about magnitudes on subsystems of the environment that are correlated with the system's pointer magnitude. Since properties not only of the system but also of subsystems of its environment are in this sense objective, it makes sense to ask whether objective claims about a system can be independently verified by observing various portions of its environment. Quantum Darwinism may now offer illuminating answers to this question by providing quantum models of interactions between a quantum system and its multipartite environment. This would be a way to show how differently situated agents can come to agree on the truth-values of significant NQMCs about a system without disturbing its state. 


\section{Conclusion}

Reflection on the paradox of Wigner's friend has persuaded some people that quantum theory cannot be understood without careful attention to the role of conscious human experience (Wigner 1967, D'Espagnat 2005). But a pragmatist interpretation (Healey 2012) permits a consistent and unambiguous treatment of the paradox without reference to consciousness. Situated agents can use quantum theory to make objective claims about the values of magnitudes in the physical world, not just about observations of them. This has helped us to predict and explain an enormous variety of otherwise puzzling physical phenomena. A true claim about the value of a physical magnitude states an objective physical fact. Ordinarily, such facts are readily independently verifiable. But acceptance of quantum theory requires one to countenance the possibility that in extraordinary circumstances information about the value of a magnitude could be irretrievably erased, making this fact no longer verifiable.

A simple magnitude claim ascribes an objective property to a physical system. But the content of this claim is a function of the environmental context. This means that the property ascribed is not intrinsic, but relational. In the case of quantum field theory, it implies that any claim about particles has a well-defined content only in some contexts, while other contexts give significance to a claim about (classical) fields. In assessing the content of a claim, an agent should consider the nature and extent of environmental delocalization of coherence. This content does not depend on the (actual or hypothetical) physical situation of the agent, even when agents base their assessments on the different quantum states appropriate to their different physical (and therefore epistemic) situations.

Science is based on observed facts, and quantum theory is no exception. According to Healey (2012), what makes quantum theory exceptional is what it teaches us about the nature of these facts. Bell $(2004,41)$ introduced the term 'beable' because he thought

it should be possible to say of a system not that such and such may be observed to be so but that such and such be so.

When making a significant claim about the value of a magnitude on a quantum system one is saying that it be so, according to Healey (2012). Perhaps this makes this magnitude a beable in Bell's sense. But Bell (2004 52) goes on to say

the beables ... can be described "in classical terms", because they are there. The beables must include the settings of switches and knobs on experimental equipment, the currents in coils, and the readings of instruments.

and (Bell 2004, 174)

The beables of the theory are those elements which might correspond to elements of reality, to things which exist. 
These passages at least suggest that acceptance of quantum theory in no way modifies the content of claims about values of magnitudes - a content that is somehow established by a fixed representation relation that obtains between language and the world (actual, or merely possible if a beable be not)! A pragmatist cannot accept such a representational account of how content accrues to a claim (Brandom 1994, 2000). According to the pragmatist interpretation of Healey (2012), to understand quantum theory one needs instead to adopt an alternative inferentialist account of what gives a claim content. By modifying the inferential relations between magnitude claims, quantum theory affects their content, rendering this contextual. By making the content of a magnitude claim about a system a function of the environment, acceptance of quantum theory cautions one against taking that claim to attribute an intrinsic property to a non-contextually existing object, even while insisting on the objectivity of the claim. This should make one think differently even about the content of everyday claims about ordinary things like the settings of switches and knobs on experimental equipment, the currents in coils, and the readings of instruments. 


\section{References}

[1] Adler, Stephen "Why decoherence has not solved the measurement problem", Studies in History and Philosophy of Modern Physics 34B (2000), pp. 135-42.

[2] Bassi, Angelo and Ghirardi, Gian Carlo (2000) "A general argument against the universal validity of the superposition principle", Physics Letters A 275, pp.373-81.

[3] Bell, John S (2004) Speakable and Unspeakable in Quantum Mechanics. Cambridge University Press, Revised Edition.

[4] Brandom, Robert (1994) Making it Explicit. Cambridge, Massachusetts: Harvard University Press.

[5] Brandom, Robert (2000) Articulating Reasons. Cambridge, Massachusetts: Harvard University Press.

[6] Brown, Harvey (1986) "The insolubility proof of the quantum measurement problem", Foundations of Physics 16, pp.857-70

[7] Brun, Todd, Finkelstein, Jerry and Mermin, N. David (2002) "How much state assignments can differ", Physical Review A 65, 032315.

[8] Bub, Jeffrey (1997) Interpreting the Quantum World. Cambridge University Press.

[9] D' Espagnat, Bernard (1990) "Toward a separable 'empirical reality'?", Foundations of Physics 20, pp. 1147-1172.

[10] D'Espagnat, Bernard (2005) "Consciousness and the Wigner's friend problem", Foundations of Physics 35, pp.1943-66.

[11] Dick, Philip K. (1968) Do Androids Dream of Electric Sheep? New York: Doubleday.

[12] Fine, Arthur (1970) "Insolubility of the quantum measurement problem", Physical Review D 2, pp.2783-87.

[13] Fine, Arthur (1982) "Joint distributions, quantum correlations, and commuting observables", Journal of Mathematical Physics 23, pp.1306-10.

[14] Gleason, Andrew (1957) "Measures on the closed subspaces of a Hilbert space", Journal of Mathematics and Mechanics 6, pp.885-93.

[15] Healey, Richard (2012) "Quantum theory: a pragmatist view", British Journal for the Philosophy of Science, doi:10.1093/bjps/axr054.

[16] Kochen, Simon and Specker, Ernst (1967) "The problem of hidden variables in quantum mechanics", Journal of Mathematics and Mechanics 17, pp.5987. 
[17] Mermin, N. David (1993) "Hidden variables and the two theorems of John Bell", Reviews of Modern Physics 65, pp.803-15.

[18] Nagel, Thomas (1986) The View from Nowhere, Oxford University Press.

[19] Ollivier, Harold, David Poulin and Wojciech Zurek (2004) "Objective properties from subjective quantum states: environment as a witness", Physical Review Letters 93, 220401.

[20] Wigner, Eugene (1967) "Remarks on the mind-body problem", in Symmetries and Reflections, Indiana University Press, Bloomington, Indiana pp.171-184. 